\title{
Sirolimus en trasplante de órgano sólido pediátrico. Experiencia en 5 casos
}

\author{
Jorge Roque E, Gloria Ríos M, Paulina Vignolo Aa, \\ Constanza Pinochet Va, Marcela Schultz, Roberto Humeres A, \\ Angela Delucchi, Montserrat Rius A ${ }^{b}$, Juan Hepp K.

\section{U se of Sirolimus in five pediatric patients undergoing solid organ transplantation}

Background: Sirolimus (SRL) is an immunosuppressive drug increasingly used in children undergoing solid organ transplantation. SRL does not cause glucose intolerance, hypertension, nephrotoxicity or neurotoxicity, offering significant potential advantages over calceneurin inhibitors (CNI). Aim: To report five children treated with SRL Material and methods: A retrospective review of four children undergoing orthotopic liver transplantation (OLT) and one undergoing renal transplantation with recurrent acute rejection (RAR), chronic rejection (CR) or toxicity due to CNI, treated with SRL between June 2001 and November 2006. Results: As primary immunosuppressive therapy, all patients received 3 drugs: CNI (Tacrolimus (FK) or Cyclosporine), mycophenolate mofetil and steroids. Mean age at treatment with SRL was 98 months. Children undergoing OLT had a late introduction of SRL (mean time after OLT: 37 months), and mean follow-up was 24 months. In this group rescue indications of SRL were RAR in one, CR in one, thrombotic thrombocytopenic purpura (TTP) in one, food allergy in one and other CNI toxicity in three. Only one did not experience adverse events due to SRL, but no one required discontinuation of SRL There were remissions of RAR, CR, TTP and food allergy. The patient with RT was switched from FK to SRL at day $18^{\text {th }}$ after RT, but he had severe neutropenia that led to discontinuation of SRL. Conclusions: SRL may be useful in pediatric solid organ transplant recipients suffering from RAR, CR, TTP, food allergy and CNI toxicity. Careful attention should be directed to detect side effects and avoid severe complications (Rev Méd Chile 2008; 136: 631-6).

(Key words: Graft rejection; Organ transplantation; Sirolimus)

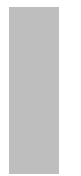

\section{Recibido el 26 de febrero, 2007. Aceptado el 3 de agosto, 2007. del Desarrollo. Santiago de Chile. \\ anterna 6 ${ }^{\underline{a}}$ año Medicina, Universidad del Desarrollo \\ ${ }^{\mathrm{b}}$ Enfermera Universitaria}

Unidad de Trasplante, Clínica Alemana de Santiago y Facultad de Medicina, Universidad

$\mathrm{L}$ a inmunosupresión tiene efectos secundarios como la diabetes mellitus (DM), enfermedad linfoproliferativa postrasplante (ELPT), neurotoxicidad y nefrotoxicidad en el caso de los inhibido-

Correspondencia a: Dr. Jorge Roque E. Avenida Manquehue 1410, piso 1, Vitacura. Fax: 2124380. E mail: jroque@alemana.cl res de los calcineurínicos (IC) o retardo del crecimiento con los esteroides ${ }^{1,2}$. Sirolimus (SRL) (rapamicina, Rapamune ${ }^{\circledR}$, Wyeth, USA) es un agente inmunosupresor del cual existe un número cada vez mayor de reportes que documentan su seguridad en trasplante en adultos y niños ${ }^{2,3}$. Ya que su mecanismo de acción y perfil de toxicidad secundaria es diferente de aquel presentado por 
los IC, ofrece potenciales ventajas sobre los esquemas inmunosupresores convencionales. Previamente reportamos un caso pediátrico de trasplante hepático ortotópico (THO) exitosamente rescatado con SRL por rechazo agudo recurrente (RAR) y toxicidad por IC $^{2}$, el objetivo actual es comunicar nuestra experiencia clínica con 5 pacientes pediátricos (trasplante renal (TR): 1 y THO: 4) en quienes usamos SRL como inmunosupresión de rescate por toxicidad, RAR y rechazo crónico (RC), así como revisar el estado actual de la literatura.

\section{Pacientes y MÉtodo}

Se revisaron las fichas clínicas de los pacientes pediátricos sometidos a THO y TR tratados con SRL entre junio de 2001 y noviembre de 2006. Los 5 pacientes recibieron al inicio una terapia triasociada con un IC (ciclosporina A (CyA), Neoral ${ }^{\circledR}$, Novartis Pharma AG, Suiza o tacrolimus (FK), Prograf ${ }^{\circledR}$, Fujisawa, USA), micofenolato mofetil (MMF) (Cell$\operatorname{cept}^{\circledR}$, Roche Pharmaceuticals, USA) y esteroides. En el seguimiento ambulatorio de los pacientes sometidos a THO se introdujo SRL cuando algún paciente presentó RAR, RC o alguna toxicidad secundaria a la inmunosupresión, haciéndose el diagnóstico de rechazo mediante biopsia revisada por el mismo patólogo. La dosis inicial de SRL utilizada era 1-2 $\mathrm{mg} / \mathrm{mt}^{2}$, sin dosis de carga, una vez al día, $4 \mathrm{~h}$ después de la dosis matinal de FK cuando se combinaban ambas drogas, buscando niveles sanguíneos de SRL y de FK entre 5-10 ng/ml. En el caso sometido a TR se cambió FK por SRL en el periodo postrasplante hospitalizado por su ausencia de nefrotoxicidad y no se realizó biopsia.

Caso 1. Niña portadora de atresia de vía biliar extrahepática (AVBE), sometida a THO a los 10 años de edad (reportado) ${ }^{2}$. Se usó SRL como terapia de rescate por RAR y DM insulina dependiente secundaria a inmunosupresión (Tabla 1). Con SRL se pudo suspender esteroides, bajar la dosis de FK $50 \%$ y no volvió a presentar rechazo agudo (RA), suspendiéndose la insulina por glicemias normales.

Caso 2. Niña con falla hepática fulminante por parvovirus B19, sometida a THO a los 7 años de edad (reportado) ${ }^{4}$. A los 19 meses post THO estando con FK 6 mg/día (nivel sanguíneo 6,8 ng/ $\mathrm{ml}$ ) y esteroides $0,25 \mathrm{mg} / \mathrm{kg} /$ día presentó $\mathrm{DM}$ insulina dependiente y RC confirmado por biopsia, (Figura 1) con bioquímica sanguínea alterada y cuadro clínico caracterizado por enflaquecimiento, hipotrofia muscular e intenso prurito. Se agregó SRL (Tabla 1) y se reactivó para nuevo THO. Evolucionó con disminución de prurito y mejoría de bioquímica sanguínea hepática. A los 2 meses de introducido el SRL presentó aumento del colesterol total a $646 \mathrm{mg} / \mathrm{dl}$, sin embargo, disminuyó a $217 \mathrm{mg} / \mathrm{dl}$ a los 13 meses y a $205 \mathrm{mg} / \mathrm{dl}$ a los 28 meses post SRL Se mantuvo asintomática y no requirió ninguna terapia para normalizar los valores de colesterol. Los triglicéridos nunca se alteraron. A los 11 meses con SRL se suspendieron los esteroides y a los 16 la insulina. A los 20 meses de tratamiento con SRL y estando con FK $5 \mathrm{mg} /$ día (nivel 2,9 ng/ml), biopsia hepática demostró resolución del rechazo crónico (Figura 2).

Caso 3. Niña portadora de AVBE sometida a THO al año y 8 meses de edad, el cual evolucionó a RC debiendo retrasplantarse (THO2) a los 2 años 11 meses de edad. Su inmunosupresión triasociada fue con CyA, presentando en los siguientes 29 meses post THO2 tres episodios de RA que respondieron a terapia con esteroides. Biopsia hepática ese mismo mes fue compatible con RC. Se cambió CyA por FK manteniendo esteroides y MMF. A los 35 meses post THO2 otra biopsia hepática confirmó persistencia de $\mathrm{RC}$, pero con bioquímica sanguínea normal. A los 54 meses post THO2 se suspendió MMF con pruebas hepáticas normales y a los 64 nueva biopsia hepática demostró ausencia de signos de RC. Al mes 68 post THO2 presentó púrpura trombocitopénico trombótico asociado al uso de FK, demostrado por actividad plasmática de metaloproteinasa ADAMS 13 de $9 \%$ y anticuerpos anti-metaloproteinasa (+) y que produjo síndrome convulsivo e insuficiencia renal aguda grave que requirió plasmaferesis, hemodiafiltración y hemodiálisis por un mes, persistiendo posteriormente con hipertensión arterial y proteinuria. Se suspendió FK e inició SRL (Tabla 1). A los 75 meses post THO2 estando con SRL y esteroides, presentó deterioro de bioquímica hepática sanguínea sospechosa de RA que no se biopsió y que normalizó con un ciclo de $10 \mathrm{mg} / \mathrm{kg} /$ día de metilprednisolona por 3 días. 
Tabla 1. Características clínicas de los pacientes tratados con Sirolimus

\begin{tabular}{|c|c|c|c|c|c|}
\hline Paciente & № 1 THO & № 2 THO & № 3 THO & №4 TR & №5 THO \\
\hline Edad inicio SRL & 149 meses & 108 meses & 115 meses & 72 meses & 45 meses \\
\hline Peso inicio SRL & 30 kilos & 30 kilos & 25 kilos & 13 kilos & 14 kilos \\
\hline \multirow{2}{*}{ Motivo de uso de SRL } & RAR & & PTT & IRA & Alergia \\
\hline & Toxicidad & Toxicidad & Toxicidad & & alimentaria \\
\hline \multirow[t]{3}{*}{ Drogas pre SRL } & $\mathrm{FK}$ & $\mathrm{FK}$ & $\mathrm{FK}$ & FK & FK \\
\hline & MMF & PRD & $\mathrm{MMF}$ & MMF & \\
\hline & PRD & & PRD & PRD & \\
\hline \multirow{5}{*}{$\begin{array}{l}\text { Efectos adversos inmuno- } \\
\text { supresión pre SRL }\end{array}$} & $\mathrm{DM}$ & $\mathrm{DM}$ & PTT & $(-)$ & Alengia \\
\hline & $\begin{array}{l}\text { Cushing } \\
\text { Sinusitis }\end{array}$ & & $\begin{array}{l}\text { Nefropatía } \\
\text { Neuropatía }\end{array}$ & & $\begin{array}{l}\text { alimentaria } \\
\text { ELPT }\end{array}$ \\
\hline & Candidiasis & & HTA & & \\
\hline & Lumbago . & & & & \\
\hline & $\begin{array}{l}\text { Osteopenia } \\
\text { Rotavirus }\end{array}$ & & & & \\
\hline $\begin{array}{l}\text { Tiempo inicio SRL } \\
\text { post Tx }\end{array}$ & 26 meses & 19 meses & 68 meses & 18 días & 34 meses \\
\hline $\begin{array}{l}\text { Tiempo seguimiento } \\
\text { post SRL }\end{array}$ & 35 meses & 28 meses & 28 meses & 15 meses & 5 meses \\
\hline Dosis SRL (mg/mt²/día) & $1-3$ & $3-5$ & $1,1-2,1$ & 1,8 & $0,8-2,5$ \\
\hline $\begin{array}{l}\text { Niveles SRL en plasma } \\
(\mathrm{ng} / \mathrm{ml})\end{array}$ & $3,4-12,5$ & $3-8,4$ & $4,2-9,2$ & $(-)$ & $2,9-4,0$ \\
\hline Inmunosupresión & SRL & SRL & SRL & FK & SRL \\
\hline post SRL & FK & FK & PRD & $\begin{array}{l}\text { MPA } \\
\text { PRD }\end{array}$ & $\mathrm{PRD}$ \\
\hline Efectos secundarios & Ulceras & Dislipidemia & Conjuntivitis & Leucopenia & $(-)$ \\
\hline atribuidos a SRL & orales & & $\begin{array}{l}\text { Edema EEII } \\
\text { Diarrea } \\
\text { Manchas } \\
\text { violáceas en } \\
\text { rostro, EESS }\end{array}$ & Neutropenia & \\
\hline
\end{tabular}

Abreviaciones: THO: trasplante hepático ortotópico; TR: trasplante renal; SRL: sirolimus; RAR: rechazo agudo recurrente; RA: rechazo agudo; RC: rechazo crónico; FK: tacrolimus; MMF: micofenolato mofetil; MPA: micofenolato sodico; PRD: esteroides; DM: diabetes mellitus; PTT: púrpura trombocitopénico trombótico; IRA: insuficiencia renal aguda; ELPT: enfermedad linfoproliferativa posttrasplante; Tx: trasplante; HTA: hipertensión arterial; EEII: extremidades inferiores; EESS: extremidades superiores.

Caso 4. Niño portador de síndrome de Cornelia de Lange con displasia renal bilateral con insuficiencia renal crónica terminal, que fue sometido a TR con donante vivo relacionado (madre) a los 6 años de edad. Evolucionó con necrosis tubular aguda permaneciendo 30 días en anuria. La inmunosupresión triasociada fue con $\mathrm{FK}$. Al día 4 postoperatorio presentó rechazo humoral agudo tratado con timoglobulina (inmunoglobulina de conejo antitimocitos humanos, Sanofi Pasteur, Francia) y plasmaferesis, descontinuando FK con buena respuesta clínica. El día 18 post TR se introdujo SRL por no ser nefrotóxico (Tabla 1) y MMF los cuales se suspendieron a las $48 \mathrm{~h}$ por leucopenia y neutropenia grave (Tabla 2). Siete días después se reinició SRL y MMF, debiendo nuevamente suspenderse a las $72 \mathrm{~h}$ por leucopenia grave. Fue dado de alta con FK, micofenolato sódico (Myfortic ${ }^{\circledR}$, Novartis Pharma AG, Suiza) y esteroides, no presentando leucopenia posterior en 15 meses de seguimiento. 


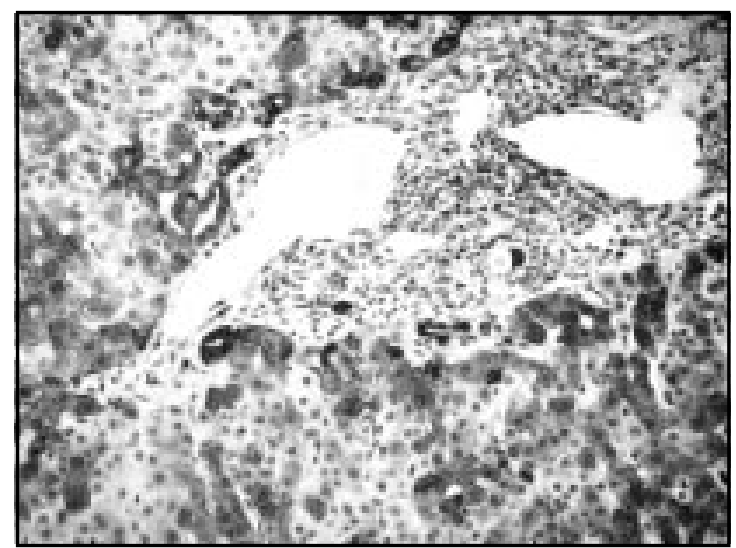

Figura 1. Espacio porta con infiltrado inflamatorio y conductillos biliares rudimentarios. A nivel de la placa limitante, proliferación seudoconductillar. (Reacción inmunohistoquímica con anticuerpos contra citoqueratinas totales, 200x).

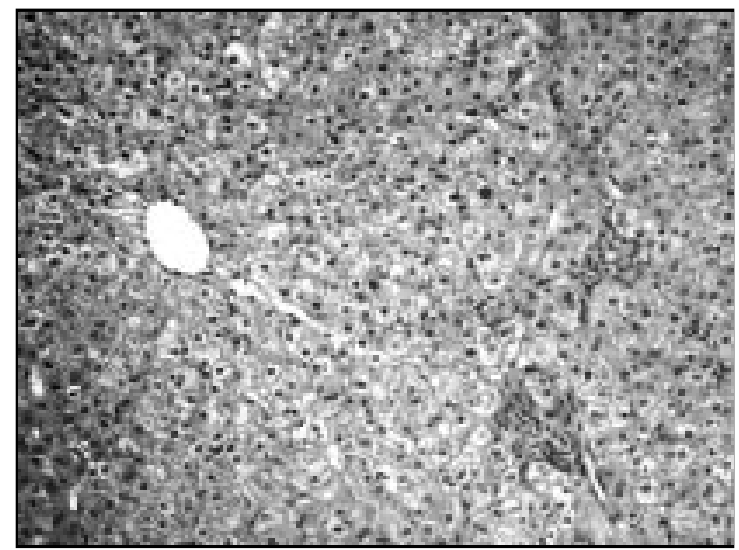

Figura 2. Espacio porta (derecha) sin infiltrado inflamatorio significativo, con conductillos normales. Zona centrolobulillar (izquierda) sin inflamación ni necrosis (hematoxilina-eosina, 200x).

Tabla 2. Leucopenia y neutropenia en paciente caso 4 tratado con Sirolimus

\begin{tabular}{|lccccc|}
\hline & $\begin{array}{c}\text { Previo } \\
\text { inicio SRL }\end{array}$ & $\begin{array}{c}\text { 2 días } \\
\text { post SRL }\end{array}$ & $\begin{array}{c}\text { Previo } \\
\text { reinicio SRL }\end{array}$ & $\begin{array}{c}\text { 3 días post } \\
\text { reinicio SRL }\end{array}$ & $\begin{array}{c}\text { 7 días post } \\
\text { reinicio SRL }\end{array}$ \\
\hline Leucocitos $/ \mathrm{mm}^{3}$ & 5.350 & 1.430 & 5.700 & 2.020 & 1.300 \\
RAN $/ \mathrm{mm}^{3}$ & 3.985 & 243 & 2.622 & 1.300 & 475 \\
\hline
\end{tabular}

SRL: Sirolimus; RAN: recuento absoluto neutrofilos

Caso 5. Niña portadora de AVBE sometida a THO a los 11 meses de edad. Al tercer mes post THO presentó ELPT por lo cual sólo se mantuvo con esteroides y tratamiento específico de su linfoma de Burkitt (reportado) ${ }^{5}$. Al mes 8 post THO, estando resuelto su ELPT presentó RA que se manejó con esteroides en dosis bajas y reintroducción de FK. A los 34 meses post THO, mediante biopsia de colon se confirmó diagnóstico de alergia alimentaria adquirida asociada al uso de FK, por lo que se suspendió este último e inició SRL (Tabla 1) con buena respuesta clínica.

\section{DisCUSIÓN}

SRL es estructuralmente similar a los IC y aunque su efecto final es el mismo, inhibir la acción de la interleukina 2, carece de acción anticalcineuríni$\mathrm{ca}^{3,6,7}$. SRL no causa nefrotoxicidad, neurotoxicidad, HTA ni $\mathrm{DM}^{1,2,7}$, por lo tanto puede permitir disminuir las dosis y los efectos adversos de los IC al hacerlo parte de la terapia inmunosupresora ${ }^{8,9}$. En los casos 1 y 2 pudo asociarse SRL al FK permitiendo disminuir las dosis y niveles sanguíneos de este último, así como su toxicidad. Los casos 3 y 5 ilustraron que en toxicidad grave por IC se pudo reemplazar FK por SRL sin mayores complicaciones para los pacientes. Sindhi y cols ${ }^{10}$ reportaron que 7 de 10 niños sometidos a trasplante tratados con FK que presentaban RAR no volvieron a presentar RA al ser introducido SRL. En el caso 1, cuando se agregó SRL al régimen inmunosupresor pudimos reducir la dosis de FK $50 \%$ y suspender los esteroides, remitiendo la DM sin RA posterior. El caso 2 pudo ilustrarnos que se 
puede revertir el $\mathrm{RC}$ con un régimen combinado de SRL y FK. En ella la mejoría clínica y bioquímica permitió suspender los esteroides antes de confirmar con biopsia la resolución del RC. Neff y cols $^{7}$ demostraron que en 13 de 21 pacientes adultos sometidos a THO con RC en terapia con FK como inmunosupresión primaria se resolvió el $\mathrm{RC}$ al agregar SRL en un periodo de 6 meses de observación, notando que el grupo respondedor tuvo mejoría bioquímica en los primeros 60 días, especialmente el descenso de la bilirrubina plasmática.

Dos de nuestros pacientes desarrollaron DM con terapia inmunosupresora basada en FK y esteroides, la cual remitió al agregar SRL. Si bien la disminución de la dosis de FK y suspensión de esteroides fue importante para descontinuar la insulina $^{2}$, hay reportes en la literatura que nos permiten especular una acción benéfica directa de SRL, el cual actuaría por medio de su unión con la proteína mammalian target of rapamycin (mTOR) inhibiendo la fosforilación y posterior degradación del receptor de insulina IRS-1 aumentando la sensibilidad a la insulina ${ }^{11,12}$ y favoreciendo el control de la glicemia sin necesidad de administrar insulina exógena.

Hay reportes de niños sometidos a THO que desarrollan alengias alimentarias asociadas al uso de FK, se postula que este tipo de toxicidad tendría etiología multifactorial, como la condición atópica del receptor y del donante o un desbalance provocado por el FK entre las subpoblaciones de linfocitos Th1 y Th2 o también puede ser consistente con una reacción mediada por IgE. Puede ocurrir hasta en $10 \%$ de los pacientes pediátricos sometidos a THO ${ }^{13-15}$. Se recomienda la suspensión del FK, con lo cual la alergia puede incluso remitir, cobrando importancia el SRL como alternativa para prevenir el rechazo. En el caso 5 hubo que reemplazar el FK por SRL, mantener con dieta hipoalergénica, revertiéndose el cuadro clínico de alergia sin presentar rechazo.

Se describe una serie de complicaciones por el uso de SRL (Tabla 3), de hecho entre $28 \%$ y $50 \%$ de los pacientes tratados con SRL debe descontinuarlo por toxicidad grave 2,3,6,16,17. En nuestra experiencia un paciente entre 5 no presentó efectos adversos por SRL y en un caso debió suspenderse SRL (asociado a MMF) por leucopenia grave. Kniepeiss y $\operatorname{cols}^{6}$ reportaron que 7 pacientes adultos sometidos a THO en quienes se usó SRL y MMF no presentaron leucopenia, mientras que Schachter y cols comunicaron que sólo 1/13 niños sometidos a TR en tratamiento con SRL y MMF presentó neutropenia grave ${ }^{17}$. En dicho caso se recomienda suspender SRL y eventual uso de factor estimulante de colonias de granulocitos ${ }^{18}$.

En conclusión SRL puede ser una droga inmunosupresora útil en niños sometidos a trasplante de órgano sólido complicados con RAR, RC, PTT, alergia alimentaria o toxicidad por IC. Sus efectos adversos deben ser cuidadosamente monitorizados para prevenir complicaciones mayores.

\section{Tabla 3. Efectos adversos descritos por uso de Sirolimus}

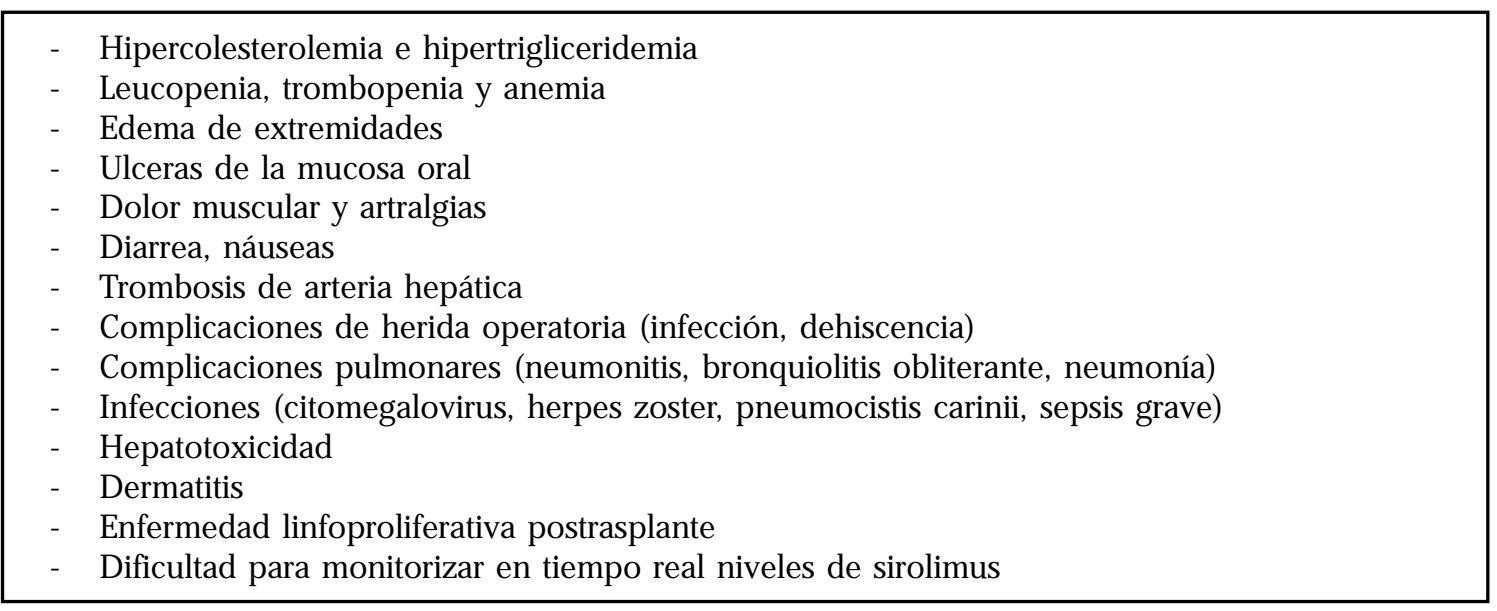




\section{REFERENCIAS}

1. Jain A, Reyes J, Kashyap R, Rohal S, Abu-Elmagd K, StaRzL T ET AL. What have we learned about primary liver transplantation under tacrolimus immunosuppression? Long Term follow-up of the first 1,000 patients. Ann Surg 1999; 230: 441-8.

2. Roque J, Ríos G, Hepp J, Humeres R, Ríos H, Herrera JM ET AL. Rescate con Sirolimus por rechazo agudo recurrente y diabetes mellitus en trasplante hepático. Caso clínico pediátrico. Rev Méd Chile 2005; 133: 1221-4.

3. KaHAN BD. The potential role of rapamycin in pediatric transplantation as observed from adult studies. Pediatr Transplant 1999; 3: 175-80.

4. Roque J, Ríos G, Hepp J, Humeres R, Ríos H, Herrera JM ET AL. Falla hepática fulminante por parvovirus B19 y trasplante hepático, caso clínico. Rev Chil Pediatr 2005; 76: 393-6.

5. Roque J, Ríos G, Humeres R, Volpi C, Herrera JM, Schultz M et AL. Early Postransplant Lymphoproliferative Disease in Pediatric Liver Transplant Recipients. Transplant Proc 2006; 38: 930-1.

6. KNiepeiss D, IBerer $F$, GRASSER $B$, SChaFFELNer $S$, TsCeLESSNIGG K. Sirolimus and mycophenolate mofetil after liver transplantation. Transpl Int 2003; 16: 504-9.

7. NefF G, Montalbano M, Slapak-Green G, Berney T, Bejarano P, Joshi A et al. A Retrospective Review of Sirolimus (Rapamune) Therapy in Orthotopic Liver Transplant Recipients Diagnosed with Chronic Rejection. Liver Transpl 2003; 9: 477-83.

8. McAlister V, Peltekian K, Malatjalian D, Colohan S, MacDonald S, Bitter-Suermann H et al. Orthotopic Liver Transplantation Using Low- Dose Tacrolimus and Sirolimus. Liver Transpl 2001; 7: 701-8.

9. Khaul RB, Houjaij A, Sawah S, El-Hout Y, MeDawWar W, Hussein M et al. Observations on Quadruple Immunosuppression Maintenance Therapy Using Rapamycin, Low-Dose Cyclosporine, Mycophenolate Mofetil, and Prednisone Following ATG Induction. Transplant Proc 2005; 37: 3031-3.
10. Sindhi $R$, Webber $\mathrm{S}$, Venkataramanan R, McGhee W, Pнiшips S, Sмith A ET al. Sirolimus for rescue and primary immunosupression in transplanted children receiving Tacrolimus. Transplantation 2001; 72: 851-5.

11. Tremblay F, Gagnon A, Veileux A, Sorisky A, MARETTE A. Activation of the mammalian tanget of Rapamycin pathway Acutely Inhibits Insulin signaling to Akt and glucose Transport in 3T3-L1 and Human Adipocytes. Endocrinology 2005; 146: 1328-37.

12. Tzatsos A, Kandror K. Nutrients Suppress Phosphatidylinositol 3-Kinase/Akt Signaling via Raptor-Dependent mTOR-Mediated Insulin Receptor Substrate 1 Phosphorylation. Mol Cell Biol 2006; 26: 63-76.

13. Ly kavieris $P$, Frauger E, Habes D, Bernard O, Debray D. Systemic FK506 and post transplant food allergy in children. Transplantation 2003; 75: 152-65.

14. Boyle RJ, Hardikar W, Tang ML. The development of food allengy after liver transplantation. Liver Transpl 2005; 11: 326-30.

15. Lacaile F, Laurent J, Bousquet J. Life-Threatening Food Allergy in a Child Treated with FK506. J Pediatr Gastroenterol Nutr 1997; 25: 228-9.

16. Dunkelberg JC, Troter JF, Wachs M, Kugelmas M, StReINBERG T, Everson GT et al. Sirolimus as primary immunosuppression in liver transplantation is not associated with hepatic artery or wound complications. Liver Transpl 2003; 9: 463-8.

17. Schachter AD, Meyers KE, Spaneas LD, Palmer JA, Salmanuliah M, Baluarte KL et al. Short Sirolimus half-life in pediatric renal transplant recipients on a calcineurin inhibitor-free protocol. Pediatr Transplant 2004; 8: 171-7.

18. Hafiz MM, Fardji RN, Froud T, Pileggi A, Baidal DA, CuRE P ET AL. Immunosuppression and procedure-related complications in 26 patients with type 1 diabetes mellitus receiving allogeneic islet cell transplantation. Transplantation 2005; 80: 1718-28. 